\title{
Analysis of a family with mitochondrial trifunctional protein deficiency caused by $H A D H A$ gene mutations
}

\author{
JINLING YANG ${ }^{1}$, DEJIAN YUAN $^{1}$, XIAOHUI TAN $^{2}$, YEXI ZENG $^{3}$, NING TANG $^{1}$,

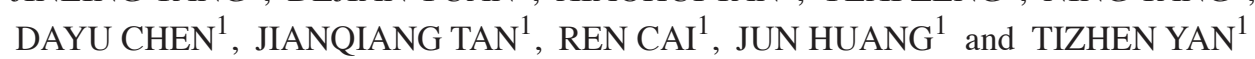

\begin{abstract}
${ }^{1}$ Newborn Screening Center, Department of Medical Genetics, Key Laboratory of Prevention and Control of Birth Defects, Liuzhou Maternity and Child Health Care Hospital, Affiliated Maternity Hospital and Affiliated Children's Hospital of Guangxi University of Science and Technology, Liuzhou, Guangxi 545000;

${ }^{2}$ School of Forensic Medicine, Southern Medical University, Guangzhou, Guangdong 510515; ${ }^{3}$ Newborn Screening Center, Huizhou Second Maternity and Child Health Care Hospital, Huizhou, Guangdong 516001, P.R. China
\end{abstract}

Received July 1, 2021; Accepted November 22, 2021

DOI: $10.3892 / \mathrm{mmr} .2021 .12563$

\begin{abstract}
Mitochondrial trifunctional protein (MTP) deficiency (MTPD; MIM 609015) is a metabolic disease of fatty acid oxidation. MTPD is an autosomal recessive disorder caused by mutations in the $H A D H A$ gene, encoding the $\alpha$-subunit of a trifunctional protease, or in the $H A D H B$ gene, encoding the $\beta$-subunit of a trifunctional protease. To the best of our knowledge, only two cases of families with MTPD due to $H A D H B$ gene mutations have been reported in China, and the $H A D H A$ gene mutation has not been reported in a Chinese family with MTPD. The present study reported the clinical characteristics and compound heterozygous $H A D H A$ gene mutations of two patients with MTPD in the Chinese population. The medical history, routine examination data, blood acyl-carnitine analysis results, results of pathological examination after autopsy and family pedigree map were collected for patients with MTPD. The HADHA gene was analyzed by Sanger sequencing or high-throughput sequencing, the pathogenicity of the newly discovered variant was interpreted by bioinformatics analysis, and the function of the mutated protein was modeled and analyzed according to 3D structure. The two patients with MTPD experienced metabolic crises and died following an infectious disease. Lactate dehydrogenase, creatine kinase (CK), CK-MB and liver enzyme abnormalities were observed in routine examinations. Tandem mass spectrometry revealed
\end{abstract}

Correspondence to: Dr Tizhen Yan, Newborn Screening Center, Department of Medical Genetics, Key Laboratory of Prevention and Control of Birth Defects, Liuzhou Maternity and Child Health Care Hospital, Affiliated Maternity Hospital and Affiliated Children's Hospital of Guangxi University of Science and Technology, 50 Boyuan Avenue, Liuzhou, Guangxi 545000, P.R. China

E-mail: yantizhen@foxmail.com

Key words: mitochondrial trifunctional protein deficiency, $H A D H A$, metabolic crisis, structural analysis that long-chain acyl-carnitine was markedly elevated in blood samples from the patients with MTPD. The autopsy results for one child revealed fat accumulation in the liver and heart. Next-generation sequencing detected compound heterozygous c.703C $>$ T (p.R235W) and c.2107G >A (p.G703R) mutations in the $H A D H A$ gene. The mother did not have acute fatty liver during pregnancy with the two patients. Using amniotic fluid prenatal diagnostic testing, the unborn child was confirmed to carry only c. $2107 \mathrm{G}>\mathrm{A}$ (p.G703R). Molecular mechanistic analysis indicated that the two variants affected the conformation of the $\alpha$-subunit of the MTP enzyme complex, and consequently affected the stability and function of the enzyme complex. The present study comprehensively analyzed the cases, including exome sequencing and protein structure analysis and, to the best of our knowledge, describes the first observation of compound heterozygous mutations in the HADHA gene underlying this disorder in China. The clinical phenotypes of the two heterozygous variants of the $H A D H A$ gene are non-lethal. The present study may improve understanding of the $H A D H A$ gene mutation spectrum and clinical phenotype in the Chinese population.

\section{Introduction}

Mitochondrial trifunctional protein (MTP) deficiency (MTPD) is a disease caused by blocked fatty acid metabolism due to defective MTP activity, and the incidence of MTPD is $1 / 140,000$ worldwide (1). MTP is an enzyme complex that catalyzes the last three steps in long-chain fatty acid metabolism, and is located in the mitochondrial inner membrane (2). MTP is an octamer composed of two distinct subunits of four $\alpha$ and four $\beta$ subunits, including three enzymes, long-chain 3-hydroxyacyl-CoA dehydrogenase (LCHAD), long-chain 2,3-enoyl-CoA hydratase (LCEH) and long-chain-3-ketothiolase (LCKAT) (3). HADHA (OMIM 600890) encodes LCHAD and LCEH, whereas $H A D H B$ (OMIM 143450) encodes LCKAT. LCHAD catalyzes the reduced dehydrogenation reaction between the $\beta$-hydroxyl group and the $\beta$-ketone group of $\mathrm{C} 12$ to $\mathrm{C} 18$ fatty acids, and NAD is used as the hydrogen 
carrier. Hydrogenases and thiolases are also specific for targeting the $\beta$-oxidation of long chain fatty acids. Enzymatic activity defects are associated with long-chain fatty acid metabolism disorders, in which long-chain fatty acids cannot be oxidized for energy supply, consequently accumulating in the cell, and causing toxic effects on the cardiac muscle, skeletal muscle and liver. The clinical manifestations of MTPD are hypoketotic hypoglycemia, rhabdomyolysis, decreased muscle tone, cardiomyopathy and liver disease, and the acute onset period can lead to death due to metabolic crisis. In routine laboratory examinations, patients show low ketotic hypoglycemia, increased creatine kinase $(\mathrm{CK})$ and elevated uric acid levels. In acyl-carnitine detection, blood samples show significant increases in multiple acyl carnitines, particularly long-chain acyl carnitine and 3-hydroxyl long-chain acyl carnitine (C12, C14, C16, C18, C14-OH, C16-OH, C18-OH and C18:1-OH), accompanied by a decrease in free carnitine $(1,4,5)$.

MTPD can involve three enzymatic defects; however, most patients have only LCHAD defects. LCHAD deficiency (LCHADD) is caused by mutations in the HADHA gene encoding LCHAD, thus causing fatty acid oxidation defects. It is divided into two types according to the gene mutation: i) Isolation LCHADD, patients carry the hotspot mutation $1528 \mathrm{G}>\mathrm{C}$, LCHAD has significantly reduced activity, whereas the integrity of MTP is not affected, and the activity of the other two enzymes is $>60 \%$; ii) LCHADD with MTPD, the coding genes of $\alpha$ and $\beta$ subunits of MTPD are mutated, and the folding stability of the multicomplex is affected, thus resulting in a decrease in activity of the three enzymes (6). The incidence of LCHADD is higher in Europe compared with in other regions, particularly in the Baltic region. For example, the incidence in Poland is 1/115,450 (7).

$H A D H A$ mutations are common in the Caucasian population, and $H A D H B$ mutations mainly exist in the East Asian population, such as in Japan, South Korea and China (8-12). Since the initial discovery of pathogenic mutations of $H A D H A$ in 1990 , most of the $59 H A D H A$ mutations reported have been frameshift mutations, nonsense mutations or alternative splicing mutations, which determine the clinical manifestations of MTPD, ranging from the life-threatening phenotype in newborns to mild rhabdomyolysis in adolescence $(6,8)$. The most common mutation of $H A D H A$ is $c .1528 \mathrm{G}>\mathrm{C}$, which results in the inactivation of LCHAD. The typical manifestations are hypoketosis, hypoglycemia, cardiomyopathy, liver disease and rhabdomyolysis. The homozygous c.1528G $>\mathrm{C}$ mutation often causes neonatal death $(5,13,14)$.

The present study reported on a Chinese family with MTPD caused by HADHA gene mutation. On the basis of clinical symptoms, biochemical testing, filter paper blood carnitine testing, autopsy and molecular detection, to the best of our knowledge, the present study was the first to identify the compound heterozygous c.703C $>$ T (p.R235W) and c.2107G $>$ A (p.G703R) mutations in $H A D H A$ associated with MTPD in China.

\section{Materials and methods}

Human participants. One boy and one girl from a southern Chinese family died suddenly for unknown reasons. The core family members were called to the Genetics
Department of Liuzhou Maternal and Child Health Hospital (Liuzhou, China) in July 2019. The clinical history of the two patients was collected and evaluated. II 2 and II 3 routine examinations, including blood biochemical indexes and filter paper blood spot acyl-carnitine testing through liquid chromatography-tandem mass spectrometry analysis, were performed. The neonatal filter paper blood spots, which had been collected at birth, were used to test acyl-carnitine and extract DNA. Collection of the two patients' urine for organic acid detection by gas chromatography-mass spectrometry was not possible because of the unexplained death of the two patients. After approval was granted by the family members, the Judicial Appraisal Center of Southern Medical University (Guangzhou, China) performed systematic anatomical and forensic pathological examinations on the body of the male patient to determine the cause of death. At that time, the mother was pregnant and expected to have a prenatal diagnosis. The present study was approved by the Ethical Committee of Liuzhou Maternity and Child Health Hospital (approval no. 2021-007) and the written informed consent was obtained from the parents.

Exome sequencing (ES) analysis. Peripheral blood anticoagulated with EDTA was collected from the parents, II1 and proband II2, and genomic DNA was extracted from peripheral blood lymphocytes. Only exome sequencing of proband II2 was performed and analyzed by AmCare Genomics Lab, and the variant detected was validated by Sanger sequencing in the whole pedigree. According to the manufacturer's instructions, genomic DNA was extracted using the SolPure Blood DNA kit (Magen Biotechnology Co., Ltd.) followed by DNA fragmentation using Q800R Sonicator (Qsonica LLC). Based on the paired-end libraries, custom designed NimbleGen SeqCap solution-based exome capture reagent (Roche NimbleGen, Inc.) was used for fragment enrichment prior to sequencing on a NextSeq500 sequencer (Illumina, Inc.). The DNA of II3, was extracted from the neonatal filter paper blood spots, which had been collected at birth, and was used for verification via Sanger sequencing. Amniotic DNA of II:4 was extracted through amniocentesis. The parents of the patients provided written informed consent for the test, and the samples of the patients were sequenced and analyzed. The genetic analysis included 1,739 genes and 25,978 coding regions containing 4,262,587 bases, with an average coverage depth of 343/-206X, with $>10 \mathrm{X}$ coverage at an interval $>99.9 \%$ and $>20 \mathrm{X}$ coverage at an interval of $99.8 \%$. All variants were filtered and annotated as follows: i) A frequency $<0.01$ in the 1000 Genomes Project (https://pubmed.ncbi.nlm.nih.gov/26432245/), ExAc (https://pubmed.ncbi.nlm.nih.gov/27899611/) and gnomAD (https://pubmed.ncbi.nlm.nih.gov/32461654/) was required; ii) mutations beyond exons and splicing sites were filtered and excluded; iii) bioinformatics prediction software was used for annotation, including the Mendelian Clinically Applicable Pathogenicity Score (https://pubmed.ncbi.nlm. nih.gov/27776117/), Combined Annotation Dependent Deletion (https://pubmed.ncbi.nlm.nih.gov/30371827/) and Polymorphism Phenotyping v2 (https://pubmed.ncbi.nlm.nih. gov/20354512/). The data from the present study have been deposited into the Sequence Read Archive (SRR16871898; https://www.ncbi.nlm.nih.gov/sra/?term=PRJNA778796). 
Sanger sequencing. The detected mutations were confirmed by Sanger sequencing in all members of the family. Total DNA of whole blood leukocytes or amniotic fluid was isolated using the QIAamp DNA Blood Mini Kit (Qiagen GmbH). Total DNA of dry blood spots was isolated by standard chloroform extraction. The primers were as follows: Forward (F) 5'-AGACCG CGTTTTCTACCTAGA-3' and reverse (R) 5'-AGGCTGACT TTATGCTTTGAGT-3' for the c.703C>T mutation; F 5'-AGA TTCTCCTTGGCCCCTTC-3' and R 5'-GTGGCTTCAGAT GGCTCTTG-3' for the c. $2107 \mathrm{G}>\mathrm{A}$ mutation. Amplification was performed using the EmeraldAmp MAX PCR Master Mix (Takara Bio USA, Inc.). PCR amplicons were sequenced on an ABI PRISM 3130 Genetic Analyzer (Applied Biosystems; Thermo Fisher Scientific, Inc.). The following thermocycling conditions were used: $95^{\circ} \mathrm{C}$ for $5 \mathrm{~min}$; followed by 35 cycles of $95^{\circ} \mathrm{C}$ for $30 \mathrm{sec}, 58^{\circ} \mathrm{C}$ for $30 \mathrm{sec}$ and $72^{\circ} \mathrm{C}$ for $60 \mathrm{sec}$; and finally $72^{\circ} \mathrm{C}$ for $7 \mathrm{~min}$.

Molecular dynamics simulation of the mutant protein. To study the effects of key amino acid mutations in HADHA on its structure, molecular dynamics simulation was used to generate virtual mutations and for structural optimization. The three-dimensional structure of wild-type HADHA (PDB ID: 5zqz) was downloaded from the RCSB Protein Data Bank (www.rcsb.org). PyMOL 2.30 (https://www.pymol.org) was used to mutate Arg235 to Trp and Gly703 to Arg.

The structure of the mutant protein was optimized by molecular dynamics simulation in AMBER16 (https://ambermd. org). The simulation temperature was set to $300 \mathrm{~K}$, and the GROMACS all-atom position and SPC water model were selected. The water molecules added around the protein formed a water box simulation system as a periodic boundary for dynamic simulation. In the simulation process, the PME algorithm was used to calculate the long-range electrostatic interactions. The integration step was set to $2 \mathrm{fs}$. Under the NVT ensemble (with the atomic number, volume and temperature kept constant), the system was balanced, and the water was optimized at $500 \mathrm{ps}$; then under the NPT ensemble (with the atomic number, pressure and temperature kept constant), the system was balanced at 500 ps. Finally, a 100 ns molecular dynamics simulation was performed.

Routine examination and blood acyl-carnitine analysis. Blood glucose, the myocardial enzyme spectrum and liver function of II 2 and II3 were analyzed during hospitalization. Neonatal blood samples of II 2 and II3 had been collected at birth, and dried blood spots of the parents and II1 were collected at the clinic visit. Acyl-carnitine content in dried blood spots was determined using the liquid chromatography tandem mass spectrometer API3200 LC-MS/MS system (Shanghai AB SCIEX Analytical Instrument Trading Co.). An API3200 triple quadrupole mass spectrometer equipped with an electrospray ionization (ESI) interface operated in positive ionization mode was used for the mass spectrometric detection. A multiple reaction monitoring mode was operated to detect specific precursor and product ions. The ESI parameters were curtain gas, 25 psi; collision gas, 6 psi; lonspray voltage, 5,500 V; temperature, $450^{\circ} \mathrm{C}$; ion source gas1, $45 \mathrm{psi}$; ion source gas 2 , $55 \mathrm{psi}$; interface heater, on. According to the ratio of index ions and the internal standard ion intensity in the mass, the relative response factor value and known internal standard concentration were used to calculate the index concentration. ChemoView ${ }^{\mathrm{TM}}(1.4 .2)$ software enabled screening of large sets of flow injection triple quadrupole mass spectrometry data for various analytes.

Pathological autopsy examination. The examination was carried out in accordance with the public safety standards of the People's Republic of China, Forensic Autopsy (GA/T147-1996), Forensic Medical Corpse Examination (GA/T149-1996), Methods of Extraction, Fixation, Packaging and Examination of Forensic Pathological Materials (GA/T148-1996) and (NYSJ-JS-BL02) of the Forensic Pathological Corpse Examination of Southern Medical University, the Instructions for the Operation of Forensic Pathological Autopsy (NYSJ-JS-BL03) of Southern Medical University, and the Instructions for the Extraction and Fixation of Forensic Pathological Materials (NYSJ-JS-BL04) of Forensic Pathology.

Through autopsy, the pathological changes observed in the organs of the patient II3 prior to death were determined, and scientific analysis and inference were performed. Subsequently, a pathological and anatomical diagnosis was reached, thus providing a theoretical basis for disease diagnosis.

\section{Results}

Clinical manifestation. The 3-year-old proband II2 was delivered at 39 weeks +2 days with a weight of 3,990 g. She was the second child of this family, and the father I1 and mother I2 denied consanguineous marriage. The proband died in the hospital after fever for half a day; sleepiness, diminished speech and progressive disturbance of consciousness for 1 day; and respiratory weakness for $30 \mathrm{~min}$. The child had shown weakness in both lower limbs and poor swallowing function. The child had been unable to walk at 1 year old and underwent surgery. The parents I1 and I2, and sister II1 had no significant clinical manifestations (Table I). Patient II3 was delivered at 38 weeks +3 days with a body weight of 3,070 g; this child died suddenly of fever and diarrhea at 7 months old, and was examined by autopsy (Table I). Routine examination analysis revealed that lactate dehydrogenase (LDH), CK, CK-MB and liver enzymes were abnormal in the past hospital examinations of proband II 2 and their younger brother II3, and the biochemical test results prior to death were the same (Table II).

Family members II 2 and II3 died because of unknown causes after symptoms including fever and diarrhea, with a high possibility of genetic disease. Proband II2 had poor walking function and poor swallowing function; II2 and II3 had findings including abnormal LDH, CK, CK-MB and liver enzyme levels, as well as hypoglycemia and liver disease. Other diseases, such as Reye syndrome, have appeared in similar clinical cases (15). Results of biochemical tests of these diseases are similar; therefore, it is important to make a differential diagnosis between them. During the last clinic visit, the physicians suspected that II 2 had a muscle-related disease, and consequently suggested a muscle biopsy and related genetic tests, which were rejected by the parents. Therefore, the reason of the disease in II 2 could not be identified. 
Table I. Clinical phenotypic and genotypic information of the affected family.

\begin{tabular}{|c|c|c|c|c|c|c|c|}
\hline ID & Sex & $\begin{array}{l}\text { Age of } \\
\text { death }\end{array}$ & $\begin{array}{l}\text { MTPD } \\
\text { state }\end{array}$ & $\begin{array}{c}\text { Inheritance } \\
\text { testing } \\
\text { method }\end{array}$ & Genotype & $\begin{array}{l}\text { Long chain } \\
\text { tandem mass } \\
\text { spectrometry } \\
\text { results }\end{array}$ & Pathological results of autopsy \\
\hline I1 & M & - & $\mathrm{U}$ & ES, Sanger & c. $703 \mathrm{C}>\mathrm{T} / \mathrm{WT}$ & $\mathrm{N}$ & - \\
\hline $\mathrm{I} 2$ & $\mathrm{~F}$ & - & $\mathrm{U}$ & ES, Sanger & c. $2107 \mathrm{G}>\mathrm{A} / \mathrm{WT}$ & $\mathrm{N}$ & - \\
\hline II1 & $\mathrm{F}$ & - & $\mathrm{U}$ & Sanger & c. $703 \mathrm{C}>\mathrm{T} / \mathrm{WT}$ & $\mathrm{N}$ & - \\
\hline II 2 & $\mathrm{~F}$ & 3 years & A & ES, Sanger & c. $703 \mathrm{C}>\mathrm{T} / \mathrm{c} .2107 \mathrm{G}>\mathrm{A}$ & $\mathrm{P}$ & - \\
\hline II3 & M & $\begin{array}{c}7 \\
\text { months }\end{array}$ & A & Sanger & c. $703 \mathrm{C}>\mathrm{T} / \mathrm{c} .2107 \mathrm{G}>\mathrm{A}$ & $\mathrm{P}$ & $\begin{array}{l}\text { Upper respiratory tract infection, } \\
\text { liver and cardiac cell fat modification }\end{array}$ \\
\hline II4 & - & - & - & Sanger & c. $2107 \mathrm{G}>\mathrm{A} / \mathrm{WT}$ & - & - \\
\hline
\end{tabular}

MTPD, mitochondrial trifunctional protein deficiency; M, male; F, female; U, unaffected; A, affected, ES, exome sequencing; WT, wild type; $\mathrm{N}$, normal (normal results); P, positive (abnormal results). - represents no relevant data.

Table II. Laboratory biochemistry findings.

\begin{tabular}{lccc}
\hline Variable & Proband II2 & Patient II3 & $\begin{array}{c}\text { Reference } \\
\text { range }\end{array}$ \\
\hline Blood biochemistry & & & \\
ALT (IU/l) & 822 & 237.4 & $0-49$ \\
AST (IU/l) & 1,103 & 595.4 & $0-49$ \\
LDH (IU/l) & 4,732 & $1,513.6$ & $109-245$ \\
CK (IU/l) & 16,448 & 5,987 & $0-200$ \\
CK-MB (IU/l) & 3,965 & 427.5 & $0-25$ \\
Glu $(\mathrm{mmol} / \mathrm{l})$ & - & 2.84 & $3.86-6.11$ \\
NT-proBNP $(\mathrm{pg} / \mathrm{ml})$ & - & $>9,000$ & $0-150$ \\
Blood spot analysis & & & \\
C0 $(\mu \mathrm{mol} / \mathrm{l})$ & 28.55 & 17.47 & $9-55$ \\
C14:1 $(\mu \mathrm{mol} / \mathrm{l})$ & 0.389 & 0.63 & $0.01-0.25$ \\
C14:2 $(\mu \mathrm{mol} / \mathrm{l})$ & 0.036 & 0.08 & $0001-0.05$ \\
C16-OH $(\mu \mathrm{mol} / \mathrm{l})$ & 0.249 & 0.33 & $0-0.06$ \\
C18:1-OH & 0.114 & 0.15 & $0-0.09$ \\
\hline
\end{tabular}

ALT, alanine aminotransferase; AST, aspartate aminotransferase; LDH, lactate dehydrogenase; CK, creatine kinase; Glu, glucose; NT-proBNP, amino-terminal type B natriuretic peptide precursor; $\mathrm{C} 0$, free carnitine; C14:1 and C14:2, long-chain acylcarnitines; $\mathrm{C} 16-\mathrm{OH}$ and $\mathrm{C} 18: 1 \mathrm{OH}$, long-chain 3-OH-acylcarnitines. - represents no relevant data.

After a systematic autopsy and forensic pathological examination of II3, death due to physical violence was excluded. Allergy-related indexes of cadaver blood from II3 were normal. These findings combined with systematic dissection and forensic pathological examination indicated no typical acute allergic pathological changes, such as clear laryngeal edema, eosinophil infiltration in the larynx and trachea submucosa, and spleen parenchyma, and the possibility of death due to acute allergy was excluded. The dissection and forensic pathology showed that II3 had extensive neutrophil infiltration in the bronchi of both lungs, diffuse inflammation of neutrophil monocytes in some alveolar cavities and edema of multiple organs. The aforementioned lesions were consistent with the pathological changes associated with typical bronchopneumonia and were serious; consequently, they were considered the cause of death for II3. Simultaneously, diffuse hepatocyte steatosis and some cardiomyocyte steatosis was detected, thus suggesting the presence of abnormal lipid metabolism or gene defects. From these results combined with the medical history analysis, it was concluded that II3 died as a cause of bronchopneumonia due to abnormal lipid metabolism.

Pathological examination showed diffuse hepatocyte steatosis and some cardiomyocyte steatosis, thus suggesting abnormal lipid metabolism or the possibility of genetic defects. Acyl-carnitine assays in past blood samples from II2 and II3 were performed and considered together with the results of the autopsy performed on II3. Acyl-carnitine analysis of the two patients revealed that the levels of $\mathrm{C} 0$ were normal, whereas those of $\mathrm{C} 14: 1, \mathrm{C} 14: 2, \mathrm{C} 16-\mathrm{OH}$ and $\mathrm{C} 18: 1-\mathrm{OH}$ were elevated (Table II). The increase in long-chain 3-OH-acyl-carnitine (C16-OH and $\mathrm{C} 18: 1-\mathrm{OH}$ ) indicated the lack of very-long-chain acyl-CoA dehydrogenase deficiency (VLCADD) or MTP.

Mutation analysis. ES of proband II2 was performed and analyzed. The compound heterozygous mutations of the $H A D H A$ gene c.703C $>$ T (p.R235W) and c.2107G $>A$ (p.G703R) were detected, and the verification of ES used Sanger sequencing (Fig. 1A). The c.2107G $>$ A variant was inherited from the father and the c. $2107 \mathrm{G}>\mathrm{A}$ variant was inherited from the mother. To date, the frequency of these two mutations is relatively low in the reference population genome database. The regions of the two mutations were confirmed to affect important components of the protein, and the amino acid sequences among species are highly conserved (Fig. 1C). The p.R235W mutation affected the second step of hydration in the four-step cycle of LCEH. Furthermore, the p.G703R mutation affected the third step of the four-step cycle of LCHAD dehydrogenation. Computer-aided analysis predicted that these two variants were highly likely to affect the structure/function of the protein. In conclusion, combined with the clinical manifestations and pedigree analysis of the patients, according to the 
A

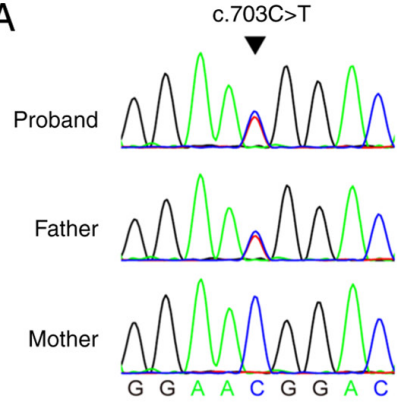

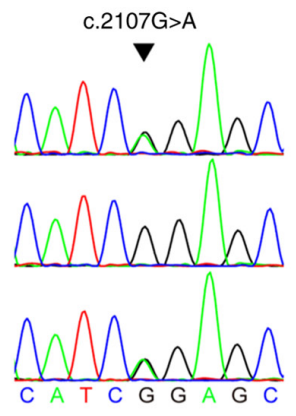

B

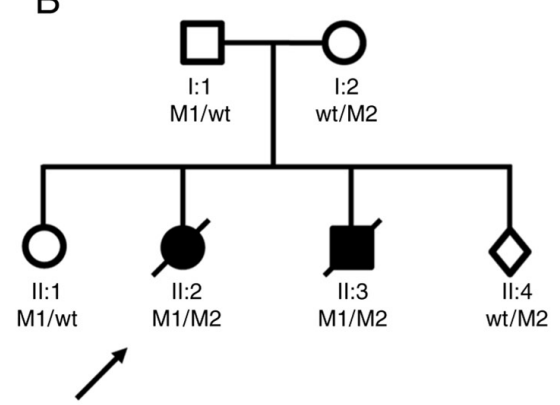

C
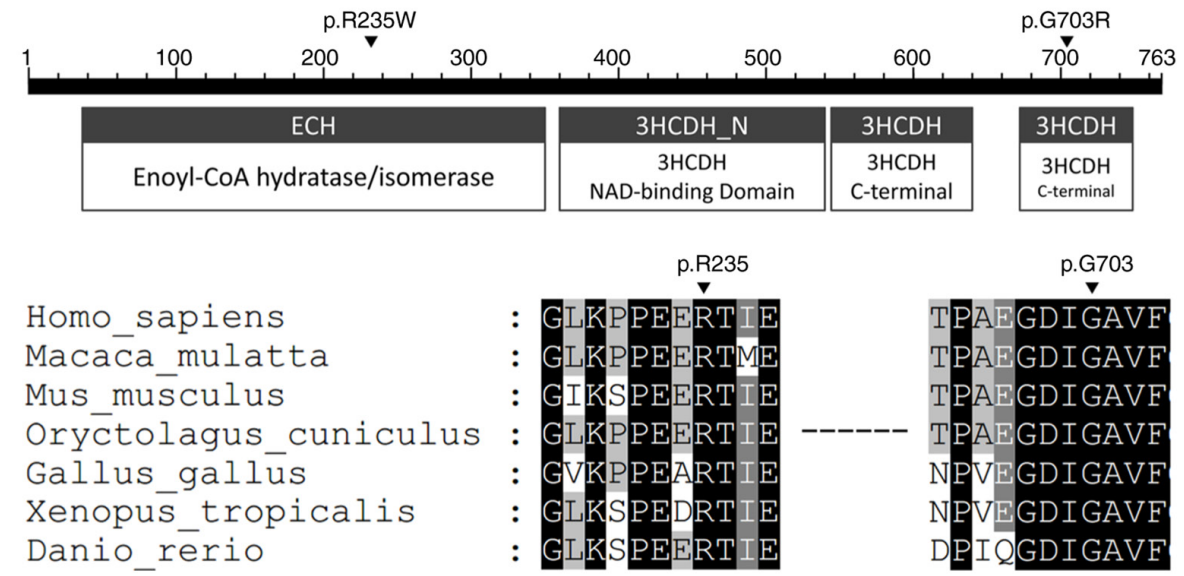

Figure 1. Genomic DNA sequencing of the pedigree. (A) HADHA compound heterozygous variants analyzed by sequencing. ES of proband II2 was performed and analyzed, and the verification of ES used Sanger sequencing. The proband II 2 had compound heterozygous c.703C $>$ T and c.2107G $>$ A mutations. The father had a heterozygous c.703C $>$ T mutation. The mother had a heterozygous c.2107G $>$ A mutation. (B) Pedigree chart. Square, male; circle, female; dark symbol, affected; arrow, proband; slash, deceased; diamond, fetus; M1, c.703C >T; M2, c.2107G>A; WT, wild type. Genotypes of II2 and II3 are both c.703C>T/c.2107G>A. (C) Conservation analysis of HADHA protein. The p.R235W mutation occurred in the enoyl-CoA hydratase/isomerase domain, and the p.G703R mutation occurred in the 3-hydroxyacyl CoA-dehydrogenase C-terminal domain. Comparison of the conservation of arginine 235 and glycine 703 among species. Black indicates $100 \%$ identity, dark grey indicates $80 \%$ identity, and gray indicates $60 \%$ identity. ES, exome sequencing.

ACMG-guideline-based variant classification (16), p.R235W and p.G703R are 'likely pathogenic.' Genetic testing of II3 also revealed the HADHA gene compound heterozygous variants c.703C $>$ T (p.R235W) and c.2107G $>$ A (p.G703R); genetic testing of the sister II1 revealed a c.703C $>$ T (p.R235W) gene mutation; and genetic testing of the fetus II4 via amniotic fluid gene testing revealed a c.2107G $>$ A (p.G703R) gene mutation (Table I; Fig. 1B). The results confirmed that the parents I1 and I2, and sister II1 were asymptomatic, and that their long chain tandem mass spectrometry results were normal. Therefore, the clinical phenotypes of the two heterozygous variants of the HADHA gene are non-lethal.

Molecular simulation and molecular mechanics. In terms of molecular characteristics, the present study demonstrated the two mutated residues were located in an essential domain of the protein that is highly conserved among species. In the mutant protein, Gly 703 was changed to Arg703 in the mutated protein, residue Arg703 formed hydrogen bond interactions with nearby Gly700, and also formed a $\pi$-cation interaction with Tyr546; Arg235 was changed to Trp235 in the mutated protein, residue Trp235 formed hydrogen bond interactions with nearby Pro231, Pro232, Tyr239 and Leu225 residues (Fig. 2A and B). The two mutations may affect the activity of the encoded enoyl-CoA hydratase/isomerase and the C-terminus of 3-hydroxyacyl-CoA-dehydrogenase, thus causing a conformational shift of other amino acids (Fig. 2C and D). The conformation of the $\alpha$ subunit of the MTPase complex could thus be destroyed, thereby affecting the stability and function of the enzyme complex. Consequently, long-chain fatty acids could not be metabolized and converted into energy.

\section{Discussion}

In the present study, ES was performed on samples obtained from a child who died of an infectious disease along with weakness in both lower limbs and swallowing dysfunction. After bioinformatics filtering and Sanger sequencing, the compound heterozygous mutations c. $703 \mathrm{C}>\mathrm{T}$ and c.2107G $>\mathrm{A}$ were identified in $H A D H A$, and the first molecular diagnosis of familial MTPD caused by HADHA gene mutations in China was determined.

In the present study, the II 2 and II 3 had compound heterozygous c. $703 \mathrm{C}>\mathrm{T}$ and c. $2107 \mathrm{G}>\mathrm{A}$ mutations, which are not common mutation sites in $H A D H A$. The p.R235W mutation affected the enoyl-CoA hydratase/isomerase domain, thus hindering the second step of hydration in the four-step cycle of LCEH. Furthermore, the p.G703R mutation affected the 3-hydroxyacyl-CoA-dehydrogenase C-terminal domain, thereby hindering the third step of the four-step cycle of LCHAD dehydrogenation. Any mutation in $H A D H B$ or $H A D H A$ may interfere with the stability of the entire MTP complex, thus increasing MTP degradation and decreasing the activity of all three enzymes (3). Among the clinical cases of mitochondrial 


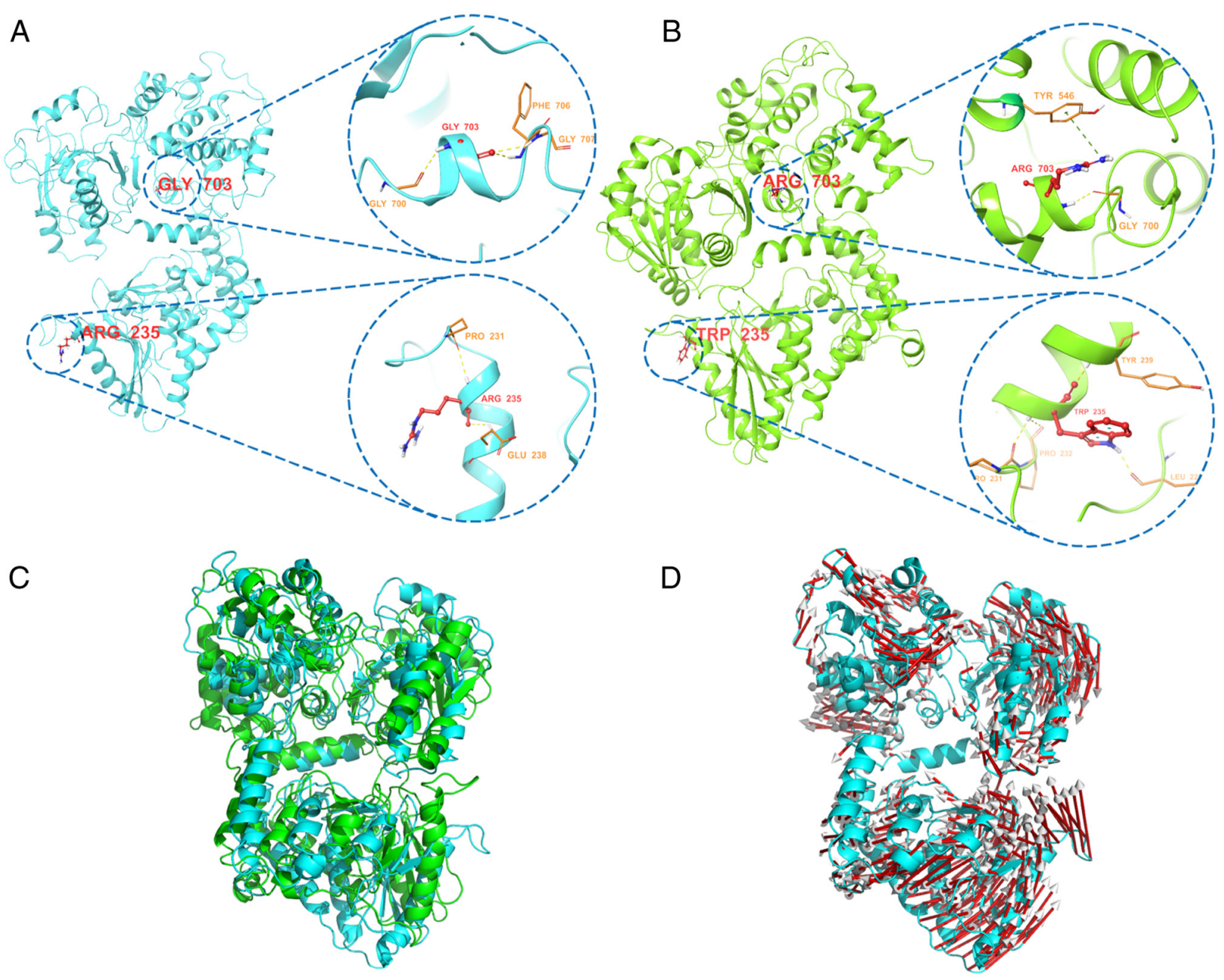

Figure 2. Molecular dynamics simulation of the mutant protein. The pre-mutated $\alpha$ subunits are in blue, and the post-mutated $\alpha$ subunits are in green. (A) Before mutation, Gly703 formed hydrogen bond interactions with the three nearby amino acids Gly700, Phe706 and Gly 707 . Arg 235 interacted with the nearby Pro231 and Glu238 amino acids, thus forming hydrogen bonds. (B) After mutation, Arg703 formed hydrogen bond interactions with the nearby Gly700 and a $\pi$-cation interaction with Tyr546. Trp235 and nearby Pro231, Pro232, Tyr239 and Leu225 formed hydrogen bond interactions. (C) Superposition simulation diagram of structural changes before and after $\alpha$ subunit mutation. (D) In the vector diagram of amino acid displacement after mutation relative to that before mutation, the red arrows indicate the direction of displacement release changes in other amino acids caused by the two mutations.

DNA deficiency, an Israeli woman has previously been reported to be homozygous for c.703C>T (p.R235W). The clinical manifestations were fatigue, fever, hypotonia, rhabdomyolysis, and a significant increase in LDH, CK and liver enzymes, in agreement with the clinical manifestations and biochemical test results in the two patients of the present study (17). The c. $2107 \mathrm{G}>\mathrm{A}$ variant has been reported in a clinical case of LCHADD; the patient was a compound heterozygote for c. $1528 \mathrm{G}>\mathrm{C}$ and c. $2107 \mathrm{G}>\mathrm{A}$, and died suddenly at the age of 7 (7). In the present study, the patients showed typical myasthenia, as well as significantly increased LDH, CK and liver enzymes, which are typical clinical features of myasthenia.

To assess the effects of p.R235W and p.G703R mutations on the structure and stability of the $\alpha$ subunit in $H A D H A$, molecular dynamics simulation was performed. Before mutation, Gly703 formed hydrogen bond interactions with the three nearby residues Gly700, Phe706 and Gly707. After mutation, Arg703 formed hydrogen bond interactions with the nearby residue Gly700 and also formed a $\pi$-cation interaction with Tyr546. The previous hydrogen bond interaction with the residues Gly700, Phe706 and Gly707 was destroyed, and the $\pi$-cation interaction formed by Tyr546 was weaker than the hydrogen bond. In wild-type HADHA, Arg235 formed hydrogen bond interactions with only two nearby residues, Pro231 and Glu238, whereas in the mutant, Trp235 formed hydrogen bond interactions with four nearby residues, Pro231, Pro232, Tyr239 and Leu225, but was unable to form a hydrogen bond with Glu238. The two mutations led to changes in hydrogen bonding, and eventually led to shifts in the positional conformation of other residues. The structure and stability of the $\alpha$ subunit was affected, and the function of the MTPase complex was destroyed, thus preventing metabolism of long-chain fatty acids and conversion into energy. Any mutation in $H A D H B$ or $H A D H A$ has been reported to potentially interfere with the stability of the entire MTP complex, thereby increasing MTP degradation and decreasing the activity of all three enzymes (3). To the best of our knowledge, no previous study has determined abnormalities in the function of specific enzymes involved in heterozygous individuals for HADHA mutations. LCHAD and LCKAT enzymatic activity in cultured fibroblasts can be assessed by skin biopsy to verify MTPase dysfunction; however, because both patients in the present 
study died, fibroblasts could not be obtained for validation. Designing molecular experiments to validate the mechanisms of these two variants in regulating the stability and function of the MTP enzyme complex will be a focus of future studies. Although our institute did not perform such assessments, we intend to cooperate with conditional laboratories to improve this deficiency.

Tandem mass spectrometry technical analysis is a technology used to detect the acyl-carnitine content in dry blood spots and blood samples. It can simultaneously detect a variety of carnitines with high sensitivity. Fatty acid $\beta$-oxidation disorder can occur in each link of fatty acids in the mitochondria and the fatty acid oxidation process. Acyl-carnitine is an intermediate of fatty acid and organic acid metabolism, and different fatty acid metabolism diseases are characterized by different acyl-carnitine profiles. Fatty acid metabolic diseases can be screened and diagnosed on the basis of changes in acyl-carnitine levels with different lengths of carbon chains (18). Tandem mass spectrometry is the gold standard analysis for the determination of carnitine concentration. The metabolite 3-OH-acyl-carnitine is usually increased during the acute phase of MTPD; however, MTPD cannot be distinguished from other fatty acid metabolic disorders (19). In the present study, neonatal screening for carnitine was not performed in the two cases (II2 and II3) at birth; however, neonatal blood samples had been collected at birth and were used after the patients' death to assess acyl-carnitine levels. Two non-acute neonatal filter paper blood samples were collected for carnitine detection. Numerous types of acyl-carnitine were revealed to be increased, particularly the C16-OH contents of II 2 and II3 were 0.249 and $0.33 \mu \mathrm{mol} / 1$, respectively (the normal reference range of $\mathrm{C} 16-\mathrm{OH}$ is $0-0.06$ $\mu \mathrm{mol} / \mathrm{l})$. Non-acute phase tandem mass spectrometry of acyl-carnitine can also indicate MTPD (20); therefore, despite the possibility of missed detection, tandem mass spectrometry remains an important first-line method for neonatal screening (1). However, it has been reported that analysis of acyl-carnitine in the blood and organic acids in the urine alone are insufficient for final diagnosis, and molecular or functional analysis is essential for the diagnosis of MTPD/LCHA (21). Notably, the enzymatic analysis of fibroblasts can be performed only in several special laboratories and cannot be performed routinely; therefore, molecular detection is the first choice for the diagnosis of patients with suspected genetic metabolic disease $(22,23)$.

In the present study, detection and analysis of ES were performed, and Sanger sequencing was used to confirm the mutation sites. In addition, pathological results of autopsies can help diagnose metabolic diseases. In the present study, diffuse steatosis of hepatocytes and partial steatosis of the myocardium were detected in the autopsy of II3, thus potentially suggesting abnormal lipid metabolism. II2 and II3 were not referred to the center of Genetic Metabolism for further diagnosis despite indications from clinical symptoms and biochemical laboratory results. The present study emphasizes the importance of confirming or eliminating the diagnosis of suspicious biochemical results and clinical symptoms in a professional center in in a timely manner. The results of the study suggested that, according to clinical symptoms, routine biochemical tests and the detection of acyl-carnitine in filter paper blood spots, patients with suspected metabolic disorders should undergo ES or functional analysis to confirm or eliminate MTPD, and rapid professional metabolic consultations are very important and helpful for early diagnosis, treatment and prevention of metabolic crisis (7).

Acute fatty liver of pregnancy (AFLP) is a rare liver disease with an incidence rate of 1/13,000 worldwide, and is associated with significant perinatal morbidity and mortality (24). In clinical practice, women with AFLP often have elevated liver enzymes and bilirubin, elevated blood ammonia, hypoglycemia, coagulation dysfunction, acute renal failure and hepatic encephalopathy. Pregnant women and their fetuses are at risk of death even if birth occurs rapidly (25). In 1991, Schoeman et al (26) first reported the relationship between AFLP and LCHAD defects, indicating that the affected women may have a genetic deficiency in a $\beta$-oxidation enzyme, thus making them prone to AFLP. In addition, Kobayashi et al (27) reported that fetal homozygous mutations in exon 13 of the $H A D H B$ gene resulted in the development of acute fatty liver in pregnant woman. In the present study, the mother was asked about any history of AFLP. All of the children were born at full-term with normal weight. It was hypothesized that although the fetus had compound heterozygous c.703C $>\mathrm{T}$ (p.R235W) and c.2107G >A (p.G703R) mutations, the possibility of AFLP in the mother was low. After identifying the pathogenic gene in the patients through familial and genetic analysis, when the mother of the child became pregnant again, amniotic fluid was collected for relevant testing. The results revealed that the fetal genotype was the same as that of the mother, who was a carrier.

In conclusion, the present study analyzed a Chinese family with MTPD caused by a HADHA gene mutation. On the basis of combined clinical symptoms, biochemical testing, filter paper blood carnitine testing, autopsy and molecular detection, to the best of our knowledge, the present study identified the first compound heterozygous c.703C $>$ T (p.R235W), c. $2107 \mathrm{G}>\mathrm{A}$ (p.G703R) mutations in $H A D H A$ associated with MTPD in China.

\section{Acknowledgements}

Not applicable.

\section{Funding}

The present study was sponsored by a grant from the Liuzhou Science and Technology Major Special Project (grant no. 2018AF10501).

\section{Availability of data and materials}

The sequencing data from the present study have been deposited into the Sequence Read Archive (SRR16871898; https://www. ncbi.nlm.nih.gov/sra/?term=PRJNA778796). The data that support the findings of this study are available from AmCare Genomics Lab but restrictions apply to the availability of these data, which were used under license for the current study, and so are not publicly available. Data are however available from the authors upon reasonable request and with permission of AmCare Genomics Lab. 


\section{Authors' contributions}

JY analyzed the data and wrote the initial draft. TY revised the draft and study design. RC and JT are pediatricians who were responsible for diagnosing and treating the children. $\mathrm{RC}$ and DY were in charge of collecting the clinical data and following up with the children. XT, YZ, NT, DC and JH performed screening by mass spectrometry and collected the data. JY and DY confirm the authenticity of all the raw data. All authors read and approved the final manuscript.

\section{Ethics approval and consent to participate statements}

The present study was approved by the Ethical Committee of Liuzhou Maternity and Child Health Hospital (approval no. 2021-007) and written informed consent was obtained from the parents.

\section{Patient consent for publication}

Not applicable.

\section{Competing interests}

The authors declare that they have no competing interests.

\section{References}

1. Lotz-Havla AS, Röschinger W, Schiergens K, Singer K, Karall D, Konstantopoulou V, Wortmann SB and Maier EM: Fatal pitfalls in newborn screening for mitochondrial trifunctional protein (MTP)/long-chain 3-Hydroxyacyl-CoA dehydrogenase (LCHAD) deficiency. Orphanet J Rare Dis 13: 122, 2018.

2. Van Vliet P, Berden AE, van Schie MK, Bakker JA, Heringhaus C, de Coo IF, Langeveld M, Schroijen MA and Arbous MS Peripheral neuropathy, episodic rhabdomyolysis, and hypoparathyroidism in a patient with mitochondrial trifunctional protein deficiency. JIMD Rep 38: 101-105, 2018.

3. Uchida Y, Izai K, Orii T and Hashimoto T: Novel fatty acid beta-oxidation enzymes in rat liver mitochondria. II. Purification and properties of enoyl-coenzyme A (CoA) hydratase/3-hydroxyacyl-CoA dehydrogenase/3-ketoacyl-CoA thiolase trifunctional protein. J Biol Chem 267: 1034-1041, 1992.

4. den Boer ME, IjlstL, Wijburg FA, Oostheim W, van Werkhoven MA, van Pampus MG, Heymans HS and Wanders RJ: Heterozygosity for the common LCHAD mutation $(1528 \mathrm{~g}>\mathrm{c})$ is not a major cause of HELLP syndrome and the prevalence of the mutation in the dutch population is low. Pediatr Res 48: 151-154, 2000.

5. Karall D, Brunner-Krainz M, Kogelnig K, Konstantopoulou V, Maier EM, Möslinger D, Plecko B, Sperl W, Volkmar B and Scholl-Bürgi S: Clinical outcome, biochemical and therapeutic follow-up in 14 Austrian patients with long-chain 3-Hydroxy Acyl CoA dehydrogenase deficiency (LCHADD). Orphanet J Rare Dis 10: $21,2015$.

6. Wanders RJ, IJlst L, van Gennip AH, Jakobs C, de Jager JP, Dorland L, van Sprang FJ and Duran M: Long-chain 3-hydroxyacyl-CoA dehydrogenase deficiency: Identification of a new inborn error of mitochondrial fatty acid beta-oxidation. J Inherit Metab Dis 13: 311-314, 1990.

7. Sykut-Cegielska J, Gradowska W, Piekutowska-Abramczuk D, Andresen BS, Olsen RK, Ołtarzewski M, Pronicki M, Pajdowska M, Bogdańska A, Jabłońska E, et al: Urgent metabolic service improves survival in long-chain 3-hydroxyacyl-CoA dehydrogenase (LCHAD) deficiency detected by symptomatic identification and pilot newborn screening. J Inherit Metab Dis 34: 185-195, 2011.

8. Boutron A, Acquaviva C, Vianey-Saban C, de Lonlay P, de Baulny HO, Guffon N, Dobbelaere D, Feillet F, Labarthe F, Lamireau D, et al: Comprehensive cDNA study and quantitative analysis of mutant HADHA and HADHB transcripts in a French cohort of 52 patients with mitochondrial trifunctional protein deficiency. Mol Genet Metab 103: 341-348, 2011.
9. Hong YB, Lee JH, Park JM, Choi YR, Hyun YS, Yoon BR, Yoo JH, Koo H, Jung SC, Chung KW and Choi BO: A compound heterozygous mutation in HADHB gene causes an axonal Charcot-Marie-tooth disease. BMC Med Genet 14: 125, 2013.

10. Fu X, Zheng F, Zhang Y, Bao X, Wang S, Yang Y and Xiong H: Mitochondrial trifunctional protein deficiency due to HADHB gene mutation in a Chinese family. Mol Genet Metab Rep 5: 80-84, 2015.

11. Bo R, Yamada K, Kobayashi H, Jamiyan P, Hasegawa Y, Taketani T, Fukuda S, Hata I, Niida Y, Shigematsu Y, et al: Clinical and molecular investigation of 14 Japanese patients with complete TFP deficiency: A comparison with caucasian cases. J Hum Genet 62: 809-814, 2017.

12. Liu ZR, Dong HL, Ma Y and Wu ZY: Identification and functional characterization of mutations within HADHB associated with mitochondrial trifunctional protein deficiency. Mitochondrion 49: 200-205, 2019.

13. Tyni T, Palotie A, Viinikka L, Valanne L, Salo MK, von Döbeln U, Jackson S, Wanders R, Venizelos $\mathrm{N}$ and Pihko H: Long-chain 3-hydroxyacyl-coenzyme A dehydrogenase deficiency with the G1528C mutation: Clinical presentation of thirteen patients. J Pediatr 130: 67-76, 1997.

14. Barvinska O, Olkhovych $\mathrm{N}$ and Gorovenko N: High prevalence of c. $1528 \mathrm{G}>\mathrm{C}$ rearrangement in patients with long chain 3-Hydroxyacyl-CoA dehydrogenase deficiency from Ukraine. Cytol Genet 52: 198-203, 2018.

15. Glasgow JF and Middleton B: Reye syndrome-insights on causation and prognosis. Arch Dis Child 85: 351-353, 2001.

16. Richards S, Aziz N, Bale S, Bick D, Das S, Gastier-Foster J, Grody WW, Hegde M, Lyon E, Spector E, et al: Standards and guidelines for the interpretation of sequence variants: A joint consensus recommendation of the American college of medical genetics and genomics and the association for molecular pathology. Genet Med 17: 405-424, 2015.

17. Scheuerman O, Wanders RJ, Waterham HR, Dubnov-Raz G and Garty BZ: Mitochondrial trifunctional protein deficiency with recurrent rhabdomyolysis. Pediatr Neurol 40: 465-467, 2009.

18. Zheng Y, Chen Y, Qiu X, Chen W, Lin Q, Zeng Y, Zhao H and Zhu W: A verification of the application of the non-derivatized mass spectrometry method in newborns screening of metabolic disorders. Medicine (Baltimore) 98: e15500, 2019.

19. Terrone G, Ruoppolo M, Brunetti-Pierri N, Cozzolino C, Scolamiero E, Parenti G, Romano A, Andria G, Salvatore F and Frisso G: Child Neurology: Recurrent rhabdomyolysis due to a fatty acid oxidation disorder. Neurology 82: e1-e4, 2014.

20. Landau YE, Waisbren SE, Chan LM and Levy HL: Long-term outcome of expanded newborn screening at Boston children's hospital: Benefits and challenges in defining true disease. J Inherit Metab Dis 40: 209-218, 2017.

21. Diebold I, Schön U, Horvath R, Schwartz O, Holinski-Feder E, Kölbel H and Abicht A: HADHA and HADHB gene associated phenotypes-identification of rare variants in a patient cohort by next generation sequencing. Mol Cell Probes 44: 14-20, 2019.

22. Pena LD, van Calcar SC, Hansen J, Edick MJ, Vockley CW, Leslie N, Cameron C, Mohsen AW, Berry SA, Arnold GL, et al: Outcomes and genotype-phenotype correlations in 52 individuals with VLCAD deficiency diagnosed by NBS and enrolled in the IBEM-IS database. Mol Genet Metab 118: 272-281, 2016.

23. Kang E, Kim YM, Kang M, Heo SH, Kim GH, Choi IH, Choi JH, Yoo HW and Lee BH: Clinical and genetic characteristics of patients with fatty acid oxidation disorders identified by newborn screening. BMC Pediatr 18: 103, 2018.

24. Ibdah JA: Acute fatty liver of pregnancy: An update on pathogenesis and clinical implications. World J Gastroenterol 12: 7397-7404, 2006.

25. Ronen J, Shaheen S, Steinberg D and Justus KR: Acute fatty liver of pregnancy:A thorough examination of a harmful obstetrical syndrome and its counterparts. Cureus 10: e2164, 2018.

26. Schoeman MN, Batey RG and Wilcken B: Recurrent acute fatty liver of pregnancy associated with a fatty-acid oxidation defect in the offspring. Gastroenterology 100: 544-548, 1991.

27. Kobayashi T, Minami S, Mitani A, Tanizaki Y,Booka M,Okutani T, Yamaguchi S and Ino K: Acute fatty liver of pregnancy associated with fetal mitochondrial trifunctional protein deficiency. J Obstet Gynaecol Res 41: 799-802, 2015.

This work is licensed under a Creative Commons Attribution-NonCommercial-NoDerivatives 4.0 International (CC BY-NC-ND 4.0) License. 